\title{
Deficiency in plasmacytoid dendritic cells and type I interferon signalling prevents diet-induced obesity and insulin resistance in mice
}

\author{
Tine D. Hannibal ${ }^{1,2}$ • Anja Schmidt-Christensen ${ }^{1}$ - Julia Nilsson ${ }^{1}$. \\ Nina Fransén-Pettersson ${ }^{1,2} \cdot$ Lisbeth Hansen ${ }^{1,2}$ • Dan Holmberg ${ }^{1,2}$
}

Received: 2 February 2017 / Accepted: 19 May 2017 /Published online: 28 June 2017

(C) The Author(s) 2017. This article is an open access publication

\begin{abstract}
Aims/hypothesis Obesity is associated with glucose intolerance and insulin resistance and is closely linked to the increasing prevalence of type 2 diabetes. In mouse models of dietinduced obesity (DIO) and type 2 diabetes, an increased fat intake results in adipose tissue expansion and the secretion of proinflammatory cytokines. The innate immune system not only plays a crucial role in obesity-associated chronic lowgrade inflammation but it is also proposed to play a role in modulating energy metabolism. However, little is known about how the modulation of metabolism by the immune system may promote increased adiposity in the early stages of increased dietary intake. Here we aimed to define the role of type I IFNs in DIO and insulin resistance.

Methods Mice lacking the receptor for IFN- $\alpha$ (IFNAR ${ }^{-/-}$) and deficient in plasmacytoid dendritic cells (pDCs) (B6.E2$2^{f l l f l}$.Itgax-cre) were fed a diet with a high fat content or normal chow. The mice were analysed in vivo and in vitro using cellular, biochemical and molecular approaches.

Results We found that the development of obesity was inhibited by an inability to respond to type I IFNs. Furthermore, the development of obesity and insulin resistance in this model was associated with pDC recruitment to the fatty tissues and liver of obese mice (a 4.3-fold and 2.7-fold increase, respectively). Finally, we demonstrated that the depletion of pDCs
\end{abstract}

Dan Holmberg

dan.holmberg@med.lu.se

1 Department of Experimental Medical Sciences, Lund University, Biomedical Center, CRC, 20502 Malmö, Sweden

2 Department of Immunology \& Microbiology, Faculty of Health and Medical Sciences, University of Copenhagen,

Copenhagen, Denmark protects mice from DIO and from developing obesityassociated metabolic complications.

Conclusions/interpretation Our results provide genetic evidence that pDCs, via type I IFNs, regulate energy metabolism and promote the development of obesity.

Keywords Adipose tissue · Obesity · pDCs · Type I interferons

$\begin{array}{ll}\text { Abbreviations } \\ \text { AT } & \text { Adipose tissue } \\ \text { BAT } & \text { Brown adipose tissue } \\ \text { DC } & \text { Dendritic cell } \\ \text { DIO } & \text { Diet-induced obesity } \\ \text { FVD } & \text { Fixable viability dye } \\ \text { HFD } & \text { High-fat diet } \\ \text { IFNAR } & \text { IFN- } \alpha \text { receptor } \\ \text { MCP-1 } & \text { Monocyte chemoattractant protein-1 } \\ \text { ND } & \text { Normal diet } \\ \text { pDC } & \text { Plasmacytoid dendritic cell } \\ \text { SAT } & \text { Subcutaneous adipose tissue } \\ \text { SVF } & \text { Stromal vascular fraction } \\ \text { TLR } & \text { Toll-like receptor } \\ \text { VAT } & \text { Visceral adipose tissue }\end{array}$

\section{Introduction}

Obesity is recognised as a medical condition characterised by the accumulation of excess body fat and is associated with a chronic state of low-grade inflammation. Low-grade inflammation in the adipose tissue (AT) involving both the adaptive and the innate immune system is reflected by a cytokineinduced acute-phase response, including elevated levels of 
TNF- $\alpha$, IL- 6 and C-reactive protein as part of an immune response of both innate and adaptive origin. A key role in the development of metabolic abnormalities has been assigned to M1-polarised macrophages that accumulate in the AT together with other immune cells [1-3].

A role for dendritic cells (DCs) in promoting macrophage infiltration to AT in obesity has been suggested [4], and an increased frequency of dysfunctional DCs in obese mice and humans supports this notion [4-6]. However, a full understanding of the role of DCs in this process is still lacking.

The Toll-like receptor (TLR)-4 (expressed on the surface of macrophages, DCs and other immune and non-immune cells) has been reported to respond to the increased levels of endogenous lipids found in obese individuals [7]. In line with this, lipid-lowering agents acting through a TLR-4-mediated mechanism can reduce the production of or the responsiveness to IFN- $\beta$ and reduce the proinflammatory actions of AT macrophages [8]. IFN- $\beta$ is a member of the type I IFN family of pleiotropic cytokines that is critical in the defence against viral infections and able to modulate both innate and adaptive immunity [9]. IFN- $\alpha / \beta$ can exert their effects by binding to their cognate receptor, the type I IFN receptor (IFNAR) complex, which stimulates the Janus kinase (JAK) signal transducer and activator of transcription (STAT) signalling pathway, leading to the transcription of several IFN-stimulated genes [10]. While antibody-mediated neutralisation of IFN- $\beta$ leads to a decrease in the mRNA expression of proinflammatory genes, including monocyte chemoattractant protein (MCP)-1, inducible nitric oxide synthase and IL- 6 , recombinant IFN- $\beta$ protein has the opposite effect [11]. In addition, IFN- $\beta$ has been reported to be necessary for maintaining the TLR-mediated MCP-1 production in macrophages [12], facilitating the recruitment of macrophages to sites of inflammation [13] and modulating the inflammatory and metabolic effects of dietinduced obesity (DIO) [14]. Thus, IFN- $\beta$ induces a feedback activation mechanism, thereby contributing to macrophagemediated inflammatory responses.

Type I IFNs are constitutively expressed in low quantities by many tissues and cells of the body in the absence of viral infection [15], supporting a potential physiological role of IFN $-\alpha / \beta$ as the initial primers of immune function as a means of maintaining immune homeostasis. A role has been proposed for type I IFN in the activation of resident macrophages and the recruitment of proinflammatory M1 macrophages to the AT and liver during obesity.

Plasmacytoid DCs (pDCs) are a unique immune cell population involved in both innate and adaptive immunity. They sense single-stranded RNA and microbial DNA through endosomal TLR-7 and TLR-9, respectively, initiating a myeloid differentiation protein 88 (MyD88)-dependent signalling cascade [16], leading to downstream activation of IRF7 and robust type I IFN responses. The pDCs are responsible for the vast majority of secreted type I IFN that promotes DC maturation, natural killer cell-mediated cytotoxicity and Th1 differentiation, and plays a crucial role in protection against viral and bacterial threats [16]. The induction and subsequent nuclear relocation of the transcription factor IRF-7 is essential for type I IFN expression in pDCs [17]. In comparison, DCs and macrophages from IRF-7 knockout mice were still able to produce normal levels of IFN- $\beta$ [18].

Studies have shown that IRF-7 deficiency prevents DIO and insulin resistance in mice [19], and that IRF-7 expression is upregulated in the arteries of obese rats [20]. In addition, murine gene expression analyses have reported significant upregulation of IFN- $\alpha / \beta$ genes in both the visceral and subcutaneous AT during obesity [21], suggesting a role for pDCderived type I IFN in obesity development. In support of this, elevated frequencies of pDCs were observed in the liver and AT of obese mice [4] and humans [22].

Here, we investigated whether DIO and the associated metabolic abnormalities are dependent on type I IFN signalling. We also analysed pDC, the major type I IFN-producing cellular subset, activity accumulated in the liver and AT during DIO and the effects of induced pDC deficiency on the development of obesity and metabolic abnormalities. We hypothesised that $\mathrm{pDCs}$, through their IFN-producing capacity, not only play significant roles in obesity-induced lowgrade inflammation and type 2 diabetes development but also in the early stages of obesity development.

\section{Methods}

Animals C57BL/6 (B6) male mice were purchased from Taconic (Ejby, Denmark). IFNAR ${ }^{-/-}$mice were kindly provided by B. Johansson-Lindbom, Lund University (Lund, Sweden). B6.E2-2 ${ }^{f l f l}$ mice were generated as previously detailed [23] and B6.Cg-Tg(Itgax-cre)1-1Reiz/J mice were purchased from Jackson Laboratory (Bar Harbor, ME, USA). B6.E2-2 $2^{f l f l}$ and B6.Cg-Tg(Itgax-cre)1-1Reiz/J mice were paired; the offspring were screened for flox-sites and crerecombinase and were bred to produce the B6.E2- $2^{f l f l}$ Itgax.cre ${ }^{+}$ and B6.E2-2 $2^{f l f l}$ Itgax.cre ${ }^{-}$mice used in this study.

B6.E2- $2^{f l f l f}$ Itgax.cre mice were bred in a specific pathogenfree animal facility at Lund University and kept under standard conditions with ad libitum access to water and food during experiments. The experiments were conducted in compliance with the National Institutes of Health guidelines, and all animal experimental procedures were approved by the ethical committee of Lund University Animal Care and Use.

Metabolic effects of DIO The mice were maintained on either a high-fat diet (HFD; $21.9 \mathrm{~kJ} / \mathrm{g}$ [5.24 kcal/g], 34.9\% (wt/wt) fat, $26.2 \%(\mathrm{wt} / \mathrm{wt})$ protein, $26.2 \%(\mathrm{wt} / \mathrm{wt})$ carbohydrate; D12492, Research Diets, New Brunswick, NJ, USA) or a normal diet (ND; $12.6 \mathrm{~kJ} / \mathrm{g}$ [3 kcal/g], 4\% (wt/wt) fat, $18.5 \%$ 
(wt/wt) protein, 55.7\% (wt/wt) carbohydrate; R36, Lactamin AB, Stockholm, Sweden) for 18 weeks, starting at 5 weeks of age.

Body weight was recorded weekly. For endpoint analysis, the animals were fasted for $6 \mathrm{~h}$ before body composition scanning using a GE-Lunar PIXImus 2 scanner (GE Healthcare, Wauwatosa, WI, USA) or an OGTT, where blood glucose was measured at various time points before and after oral glucose administration $(2 \mathrm{~g} / \mathrm{kg})$. Cheek blood samples were collected before and after glucose administration for hormone analysis. The mice were anaesthetised with Hypnorm/Dormicum (Vetapharma, Leeds, UK/Roche, Basel, Switzerland) and transcardially perfused with $15 \mathrm{ml}$ PBS, followed by dissection of subcutaneous white adipose tissue (SAT), visceral white adipose tissue (VAT), brown adipose tissue (BAT), the spleen and the liver for flow cytometry.

Flow cytometric analysis Single cell suspensions from the spleen were obtained by disrupting the tissue through a $70 \mu \mathrm{m}$ cell strainer. The livers were mechanically minced into small $>1 \mathrm{~mm}$ pieces, washed twice in PBS, treated with collagenase II (Invitrogen, Carlsbad, CA, USA) for $40 \mathrm{~min}$ at $37^{\circ} \mathrm{C}$, and single cells were suspended through a $70 \mu \mathrm{m}$ cell strainer and separated by 50/30 Percoll (GE Healthcare) gradient centrifugation. The white adipose tissue samples were mechanically minced and collagenase II digestion was performed for $40 \mathrm{~min}$ at $37^{\circ} \mathrm{C}$ to fractionate the AT into adipocytes and a stromal vascular fraction (SVF). The SVF was resuspended in HEPES-buffered RPMI medium (Gibco, Waltham, MA, USA) and single cells were suspended through a $70 \mu \mathrm{m}$ cell strainer. All samples were resuspended in FACS buffer (PBS, $3 \%$ [vol./vol.] FCS, 2 mmol/1 EDTA) prior to surface staining, preincubated with anti-CD16/32 (Fc 'blocking' antibody, clone 2.4G2) (BD Biosciences, San Jose, CA, USA) for $15 \mathrm{~min}$ at $4^{\circ} \mathrm{C}$ and subsequently stained with fluorescentlabelled primary antibodies for $25 \mathrm{~min}$ at $4^{\circ} \mathrm{C}$. The following antibodies were used: anti-TCR $\beta$ (clone H57-597) and antiSiglecF (clone E50-2440) from BD Biosciences; anti-CD317 (clone 120G8.04) from Dendritics (Lyon, France); anti-Ly6G (clone 1A8), anti-Ly6C (clone HK1.4), anti-NK1.1 (clone PK136), anti-CD45 (clone 30-F11), anti-I-A/I-E (clone M5/ 114.15.2), anti-CD11b (clone M1/70) and anti-CD206 (clone C068C2) from Biolegend (San Diego, CA, USA); and antiCD4 (clone GK1.5), anti-F4/80 (clone BM8), antiCD45R(B220) (clone RA3-6B2), anti-CD8 (clone 53-6.7), anti-CD11c (clone N418), anti-SiglecH (clone 440c), antiCD19 (clone 1D3) and fixable viability dye (FVD) from eBioscience (Santa Clara, CA, USA). The cells were fixed using the Foxp3 Staining Buffer Set (eBioscience). Samples containing biotin-coupled antibodies were incubated for 25 min at $4^{\circ} \mathrm{C}$ with secondary Streptavidin-PECF594 antibodies (BD Biosciences). Cells were washed twice in FACS buffer and data acquisition was performed on a BD LSR II flow cytometer with FACSDiva software (BD Biosciences). A minimum of $50,000 \mathrm{CD}^{2} 5^{+}$events were analysed for each sample. The analysis was performed using the FlowJo software (FlowJo LLC, Ashland, OR, USA).

Insulin ELISA Cheek blood samples were collected from mice as a part of the OGTT, and plasma was isolated with the aid of EDTA-K tubes (Sarstedt, Nümbrecht, Germany). The tubes were spun at $1000 \times g$ for $15 \mathrm{~min}$ to isolate plasma. Insulin levels were analysed using an Ultra Sensitive Mouse Insulin ELISA kit (CrystalChem, Downers Grove, IL, USA).

Statistical analysis Pairwise group comparison was performed using unpaired two-tailed Student's $t$ test or two-way ANOVA using GraphPad Prism 5 (GraphPad Software, San Diego, CA, USA). The values are presented as the mean $\pm \mathrm{SD}$.

\section{Results}

Development of DIO and insulin resistance is dependent on type I IFN signalling To gain insight into the potential role of type I IFN in obesity and obesity-related metabolic abnormalities, we used the HFD mouse model of type 2 diabetes. From 5 weeks of age, male C57BL/6 (B6) mice were given ad libitum access to either an HFD or ND for 18 weeks. As shown before [24], mice fed an HFD showed a significant weight increase of $64 \%$ compared with ND-fed littermates (Fig. 1a). At the 18 week endpoint, the obese mice had developed glucose intolerance and insulin resistance (Fig. 1b, c). The detrimental stage of metabolic disease was evident by the increase in the sizes of the fat pads and the liver, which were significantly enlarged in obese mice (HFD, $2.35 \pm 0.29 \mathrm{~g}$; ND, $1.10 \pm 0.16 \mathrm{~g}$ ) (Fig. 1d). Using an x-ray densitometer, lean and fat mass was compared between the two groups, by which the difference in weight gain was attributed to a significant increase in fat mass (Fig. 1e, f).

To directly test the hypothesis that type I IFN plays a significant role in the development of obesity and type 2 diabetes-related metabolic abnormalities, we exposed mice lacking the receptor for IFN- $\alpha$ (IFNAR ${ }^{-1-}$ ) to an HFD. In support of the hypothesis, we found that the HFD-fed IFNAR $^{-/-}$mice displayed a relative resistance to dietinduced weight gain compared with the B6 control mice on an HFD (Fig. 1g). This difference was significant after 6 weeks following the initiation of HFD feeding. At the endpoint after 18 weeks of HFD, the IFNAR ${ }^{-/}$mice had an $11 \%$ lower body weight compared with the control B6 mice (IFNAR ${ }^{-/}$HFD $44.7 \mathrm{~g}$ vs B6 HFD $49.9 \mathrm{~g}$ ). This was associated with significantly smaller subcutaneous fat depots and a lower total fat mass in the HFD-fed IFNAR ${ }^{-/-}$mice compared with B6 mice (Fig. 1f, h). 

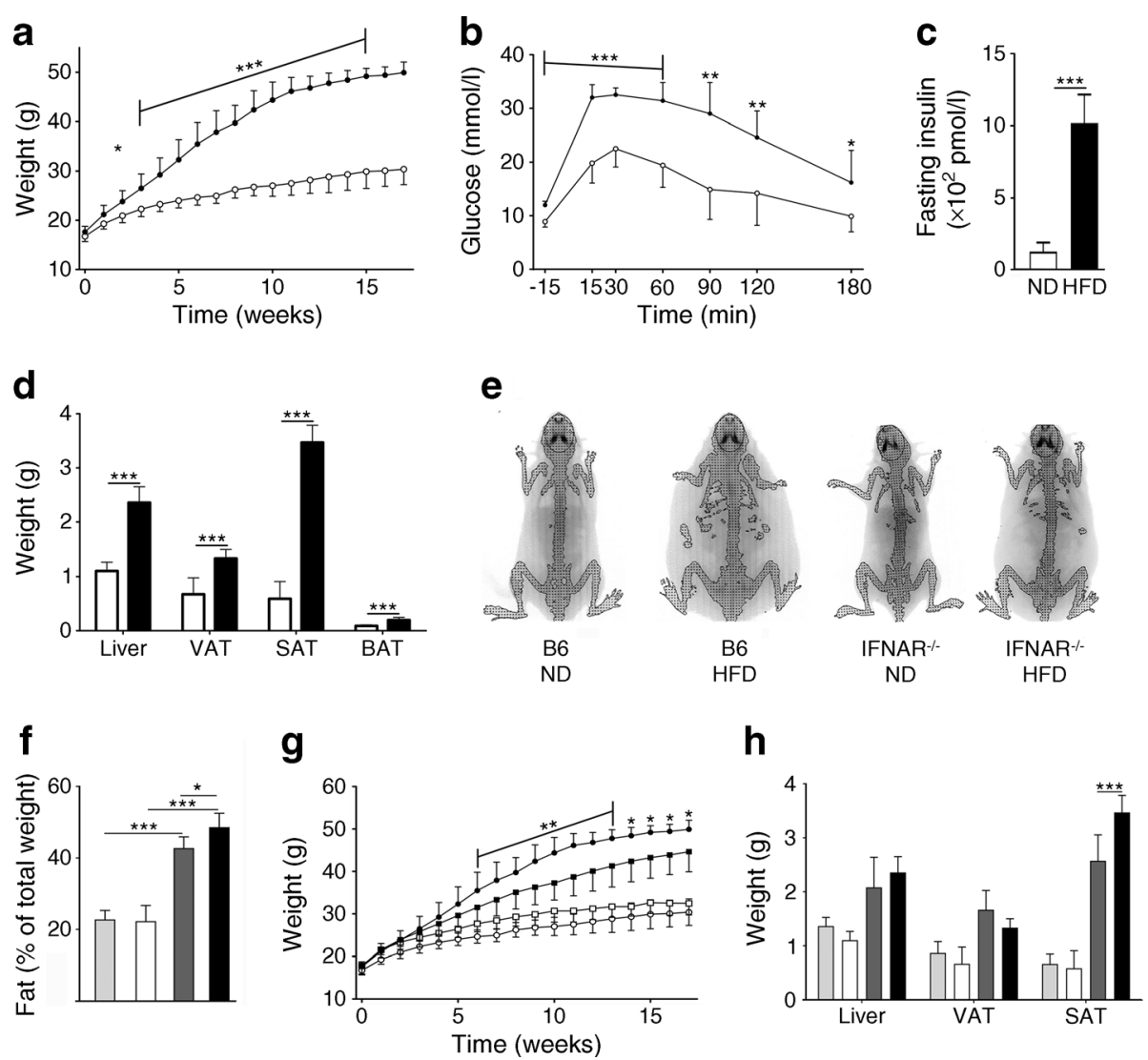

Fig. 1 Characteristics and metabolic profile of B6 and IFNAR ${ }^{--}$male mice exposed to ND or HFD. Mice were given an ND or HFD for 18 weeks, starting at 5 weeks of age. (a) Weekly weight measurements of the B6 mice fed an ND (white circles) or HFD (black circles). (b) An OGTT was performed after 18 weeks. B6 mice were given an oral dose of glucose and blood glucose was determined from tail vein samples at different time points. ND, white circles; HFD, black circles. (c) Fasting insulin levels in ND- or HFD-fed B6 mice. (d) Individual tissue weights from B6 mice after 18 weeks. ND, white bars; HFD, black bars. (e) Image of an X-ray scan determining the fat content in $\mathrm{B} 6$ and $\mathrm{IFNAR}^{-/}$mice after 18 weeks on an ND or HFD. (f) Proportion of fat in B6 and IFNAR ${ }^{-/-}$

DIO led to elevated numbers of proinflammatory macrophages in AT which are reduced in absence of IFNAR1 Obese mice developed a low-grade inflammation in the visceral fat deposits, with an increased number of leucocytes and an altered immune cell repertoire characterised by elevated levels of $\mathrm{CD}_{11 \mathrm{c}^{+}}$proinflammatory macrophages $\left(\mathrm{CD} 11 \mathrm{c}^{+} \mathrm{CD} 11 \mathrm{~b}^{+} \mathrm{F} 4 / 80^{+}\right)$(Fig. 2a-d).

When examining the infiltrating cell population in the inflamed AT, we found an expectedly large proportion of the $\mathrm{CD}_{11 \mathrm{c}^{+}}$cells to be proinflammatory macrophages (F4/ $80^{+} \mathrm{CD} 11 \mathrm{~b}^{+} \mathrm{CD} 11 \mathrm{c}^{+}$) (Fig. $\left.2 \mathrm{~b}-\mathrm{d}\right)$. In the liver the number of proinflammatory macrophages was also found to be significantly increased (Fig. 2c). When applying a subsequent analysis of the total $\mathrm{CD} 11 \mathrm{c}^{+}$population in the liver, we found significantly higher numbers in the obese state but also noted that a large proportion of these cells could not be accounted for by the macrophage population (Fig. 2c). mice after 18 weeks of an ND or HFD determined by x-ray scanning. (g) Weekly weight measurements of the IFNAR ${ }^{-/-}$(squares) and control B6 (circles) mice fed an ND (white symbols) or HFD (black symbols). A significant difference was only indicated for B6 mice on an HFD. (h) Individual tissue weights of IFNAR ${ }^{--}$and B6 mice after 18 weeks. (f, h) IFNAR $^{-/-} \mathrm{ND}$, light grey bars; IFNAR ${ }^{-/}$HFD, dark grey bars; B6 ND, white bars; B6 HFD, black bars. $n=6$ for ND- and $n=18$ for HFD-fed animals, except for in $(\mathbf{b}, \mathbf{c})$ where $n=6$ for ND- and HFD-fed animals. The experiments were repeated independently $2-3$ times. $* p \leq 0.05$, $* * p \leq 0.01, * * * p \leq 0.001$ using two-way ANOVA $(\mathbf{a}, \mathbf{b}, \mathbf{f}, \mathbf{g}, \mathbf{h})$ or Student's $t$ test $(\mathbf{c}, \mathbf{d})$

To determine whether the metabolic effects observed in the absence of type I IFN signalling were linked to a decreased inflammation in VAT and the liver we exposed the IFNAR ${ }^{-/}$ mice and B6 control mice to an 18 week period of HFD feeding and compared them to groups fed an ND (Fig. 2eg). We observed that the accumulation of proinflammatory macrophages in VAT of B6 mice was reduced in the absence of IFNAR1 (Fig. 2g). This would be in line with a previously suggested role of IFN-I in proinflammatory polarisation of VAT-recruited macrophages [22].

DIO led to elevated numbers of pDCs Because it has been previously reported that $\mathrm{pDCs}$ increase in both the liver and AT of HFD-treated animals [4], we hypothesised that this DC subset could account for the observed increase in $\mathrm{CD} 11 \mathrm{c}^{+}$ cells in the liver. We therefore identified the pDCs among the $\mathrm{CD} 11 \mathrm{c}^{+}$population by gating away $\mathrm{B}$ cells $\left(\mathrm{CD} 19^{+}\right)$, 

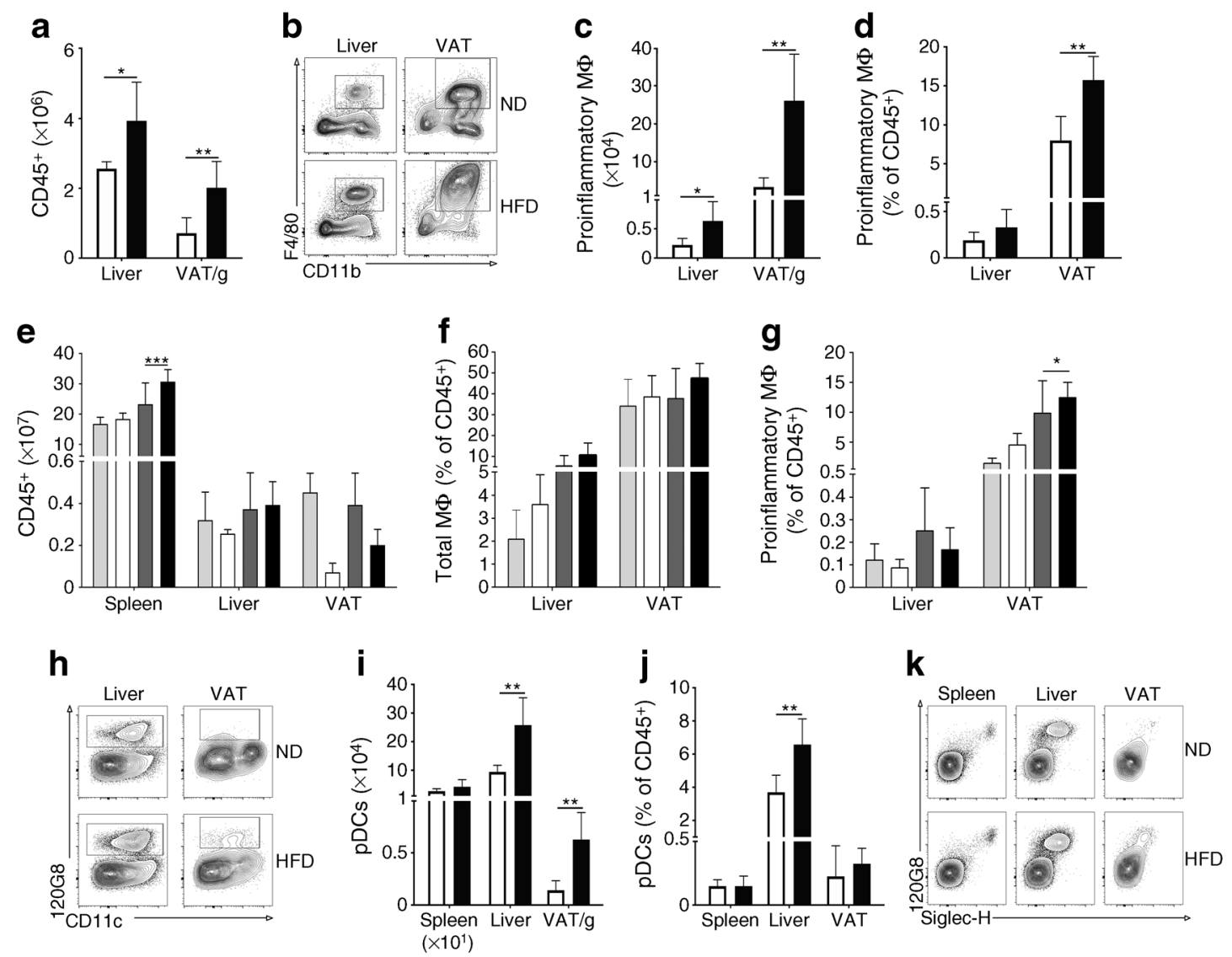

Fig. 2 DIO promotes proinflammatory macrophage and $\mathrm{pDC}$ infiltration in the AT and liver. Flow cytometry analysis of leucocytes from the spleen and liver and SVF from the VAT of lean (white bars) and obese (black bars) B6 mice or lean (light grey bars) and obese (dark grey bars) IFNAR $^{-/}$mice. (a) Number of live CD $45^{+}$cells. (b) Representative contour plots of $\mathrm{FVD}^{-} \mathrm{CD} 45^{+} \mathrm{CD} 11 \mathrm{c}^{+} \mathrm{F} 4 / 80^{+} \mathrm{CD} 11 \mathrm{~b}^{+}$cells from the liver or VAT of lean (ND) and obese (HFD) B6 mice with the gating for the proinflammatory macrophage subset of total $\mathrm{CD}^{+} 5^{+}$cells shown. (c) Number and (d) percentage of $\mathrm{FVD}^{-} \mathrm{CD} 45^{+} \mathrm{CD} 11 \mathrm{c}^{+} \mathrm{F} 4 / 80^{+} \mathrm{CD} 11 \mathrm{~b}^{+}$proinflammatory macrophages out of $\mathrm{CD} 5^{+}$cells. (e) Number of live $\mathrm{CD} 45^{+}$cells. (f) Percentage of total $\mathrm{FVD}^{-} \mathrm{CD} 45^{+} \mathrm{CD} 11 \mathrm{~b}^{+} \mathrm{F} 4 / 80^{+}$

macrophages out of live $\mathrm{CD} 45^{+}$cells. (g) Percentage of $\mathrm{FVD}^{-} \mathrm{CD} 45^{+} \mathrm{CD} 11 \mathrm{c}^{+} \mathrm{F} 4 / 80^{+} \mathrm{CD} 11 \mathrm{~b}^{+}$proinflammatory macrophages out of live CD $45^{+}$cells. (h) Representative contour plots of $\mathrm{FVD}^{-} \mathrm{CD} 45^{+} \mathrm{CD} 19^{-} \mathrm{B} 220^{+} 120 \mathrm{G} 8^{+} \mathrm{CD} 11 \mathrm{c}^{\text {int }}$ cells from the liver or VAT of lean (ND) and obese (HFD) B6 mice with the gating for the pDC subset of total live $\mathrm{CD} 45^{+}$cells shown. (i) Number and (j) percentage of pDCs out of live CD45 $5^{+}$cells. (k) Representative contour plots of $\mathrm{FVD}^{-} \mathrm{CD} 45^{+} \mathrm{CD} 19^{-} \mathrm{B} 220^{+} \mathrm{CD} 11 \mathrm{c}^{\text {int }}$ cells showing the expression of Siglec-H and 120G8. $n=6$ in all groups of animals. The experiments were repeated independently at least 2 times. ${ }^{*} p \leq 0.05, * * p \leq 0.01$, $* * * p \leq 0.001$ using Student's $t$ test. M $\Phi$, macrophage

natural killer cells $\left(\mathrm{NK} 1.1^{+}\right)$and macrophages $\left(\mathrm{F} 4 / 80^{+}\right.$and $\mathrm{CD} 11 \mathrm{~b}^{+}$) and focusing on the $\mathrm{B} 220^{+} 120 \mathrm{G} 8^{+} \mathrm{CD} 11 \mathrm{c}^{(\mathrm{int})}$ subset (Fig. $2 \mathrm{~h}-\mathrm{j}$ ). While the expression of the pDC marker Siglec-H was high in the splenic pDC population, the same subset in the liver and fat depots demonstrated variable expression profiles (Fig. 2k); the marker itself was also recently found to be less specific than initially proposed [25] and is partly sensitive to collagenase treatment [26]. The 120G8 antibody specifically targets BST-2 and constitutes a suitable marker for pDCs, as illustrated by its ability to eliminate pDCs through antibody depletion without significantly affecting other cell populations [27]. Using this gating strategy, we found that the pDC population was significantly increased in both the liver and VAT after HFD feeding (a 2.7-fold increase in the liver and 4.3-fold increase in VAT), and was most abundant in the liver (Fig. 2j). In the liver, pDCs accounted for almost $50 \%$ of the CD11 $\mathrm{c}^{+}$

population in the obese group and approximately $30 \%$ in the lean group (Fig. $2 \mathrm{~h}-\mathrm{j}$ ).

pDC deficiency protects against weight gain The observed accumulation of pDCs in both the liver and AT, as well as the protection from obesity development in the IFNAR knockout mice, suggest that this cellular subset could play a role in the pathology observed in the DIO model. To directly address this issue, we used the B6.E2-2 $2^{f l f l}$.Itgax-cre mouse strain, in which the conditional knockout of E2-2 results in a specific block of the development of pDCs [23] (Fig. 3). A pronounced decrease in this subset is evident in both the bone marrow [23] and peripheral organs, including the spleen, liver and AT (Fig. 3a, b). In contrast, no significant alteration was seen in other haematopoietic subsets, such as macrophages and DCs (Fig. 3c-e). 

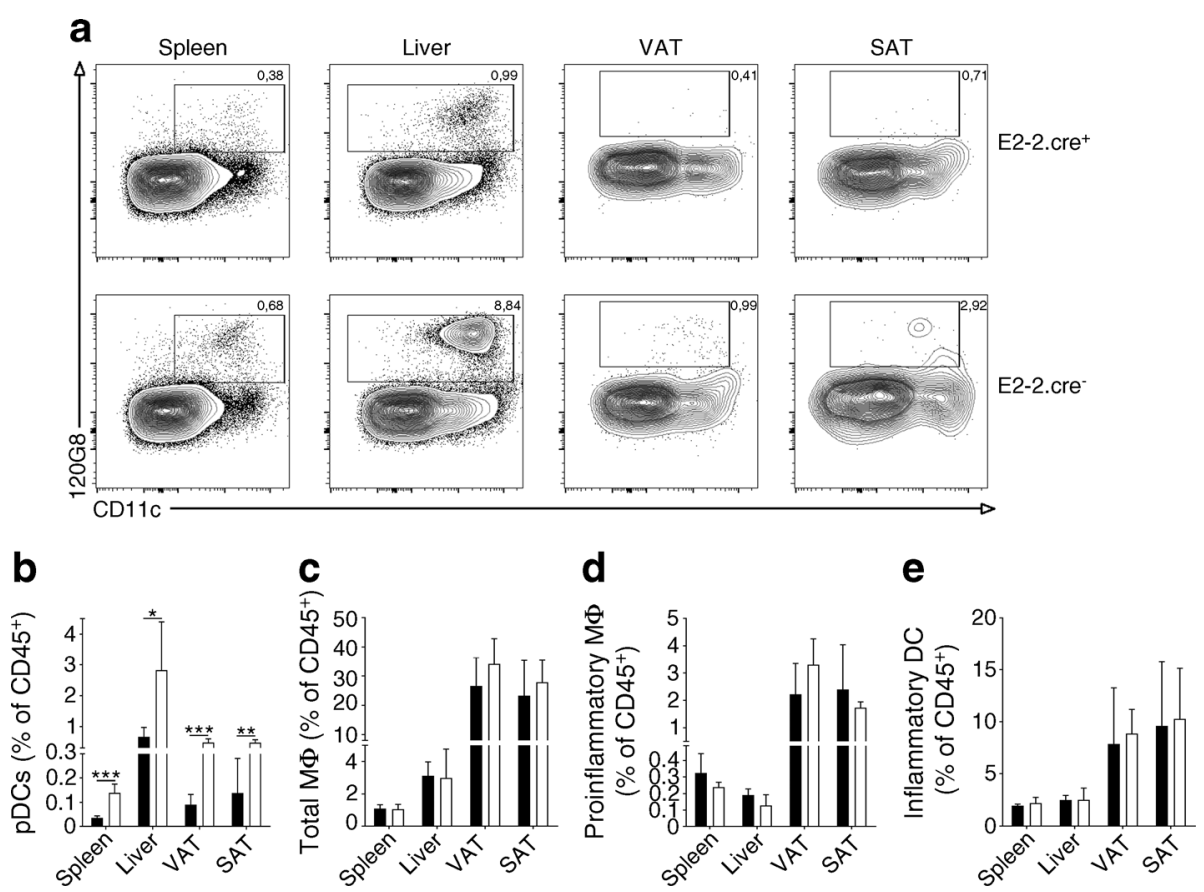

Fig. 3 Significant reduction in $\mathrm{pDCs}$ in the E2-2.cre ${ }^{+}$mouse. Flow cytometry analysis of $\mathrm{CD} 45^{+}$leukocytes from the spleen and liver and SVF from the VAT and SAT of E2-2.cre ${ }^{+}$and E2-2.cre ${ }^{-}$mice. (a) Representative contour plots of $\mathrm{FVD}^{-} \mathrm{CD} 45^{+} \mathrm{CD} 19^{-} \mathrm{B} 220^{+} 120 \mathrm{G}^{+} \mathrm{CD} 11 \mathrm{c}^{\text {int }}$ cells with gating for pDCs. Percentages of (b) pDCs and (c) total $\mathrm{FVD}^{-} \mathrm{CD} 45^{+} \mathrm{CD} 11 \mathrm{~b}^{+} \mathrm{F} 4 / 80^{+}$ macrophages. (d) $\mathrm{FVD}^{-} \mathrm{CD} 45^{+} \mathrm{CD} 11 \mathrm{c}^{+} \mathrm{F} 4 / 80^{+} \mathrm{CD} 11 \mathrm{~b}^{+}$proinflammatory

Next we exposed E2-2 conditional knockout (E2-2.cre $\left.{ }^{+}\right)$ mice and control littermates (E2-2.cre ${ }^{-}$) to an 18 week period of HFD feeding and compared them to groups fed an ND. As with the IFNAR ${ }^{-/}$mice, we observed a significant difference in weight gain between the E2-2.cre ${ }^{+}$and the E2-2.cre groups, with a significantly lower weight gain in the E22.cre ${ }^{+}$mice (45.6 $\mathrm{g}$ for E2-2.cre ${ }^{+}$and $54.4 \mathrm{~g}$ for E2-2.cre ${ }^{-}$mice) that was evident from as early as week 4 on an HFD (Fig. 4a). The reduced weight gain was accompanied by increased insulin sensitivity (Fig. 4b). Thus, while the control group at the endpoint had reached the level of insulin resistance, the E22.cre ${ }^{+}$mice were protected from developing insulin resistance (Fig. 4b). An improved, albeit not significant, GTT was also noted (Fig. 4c).

Interruption of type I IFN signalling and pDC deficiency prevents DIO and insulin resistance by affecting energy metabolism To investigate if the observed protection from weight gain among the IFNAR ${ }^{-/}$mice was the result of a decrease in energy intake or energy expenditure, we registered the food intake throughout the experiment. As illustrated in Fig. 5a, no significant difference between the IFNAR ${ }^{--}$and B6 mice was observed at any time point, suggesting that the observed difference in weight gain was due to different energy expenditure. Similarly, when the metabolic differences between E2-2.cre ${ }^{+}$and E2-2.cre ${ }^{-}$mice were assessed through

macrophages. (e) $\mathrm{FVD}^{-} \mathrm{CD} 45^{+} \mathrm{CD} 11 \mathrm{c}^{\mathrm{hi}} \mathrm{CD} 11 \mathrm{~b} \mathrm{MHCII}^{+} \mathrm{DCs}$ and in the spleen, liver, VAT and SAT of E2-2.cre ${ }^{+}$(black bars) and E2-2.cre ${ }^{-}$(white bars) mice. (b-e). $n=6$ in each mouse group. The experiments were repeated independently at least 2 times. $* p \leq 0.05, * * p \leq 0.01$, *** $p \leq 0.001$ using Student's $t$ test. M $\Phi$, macrophage

their food intake, no significant difference was observed (Fig. 5b). Additionally, when assessing the mice, which were kept in groups of 4-6 individuals, no differences in behavioural patterns were seen between the groups. These results suggest that the ablation of type I IFN signalling, as well as pDC deficiency, prevented the mice from developing DIO and insulin resistance by affecting energy metabolism.

\section{Discussion}

In the current study, we present evidence that type I IFN plays a critical role in the development of obesity and diabetes in the DIO mouse model of type 2 diabetes. Using IFNAR ${ }^{-1}$ mice, we demonstrated that the absence of type I IFN signalling protected them from the development of DIO and diabetes. Since pDCs, a major source of type I IFN [16, 28], have been reported to accumulate in the AT and liver of obese mice [4], we reasoned that this cellular subset could constitute a key component in the type I IFN-mediated infiltration of proinflammatory M1 macrophages during obesity development. In agreement with this hypothesis, we demonstrated that $\mathrm{pDC}$-deficient E22. cre $^{+}$mice displayed a similar resistance to developing DIO and insulin resistance as observed in $\mathrm{IFNAR}^{-/-}$mice.

Type I IFNs are prominent in viral infections, where they promote host defence mechanisms. However, a role of the 


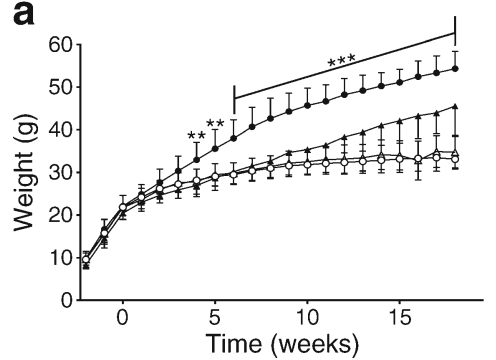

Fig. 4 Characteristics and metabolic profile of E2-2.cre ${ }^{+}$and E2-2.cre mice on ND or HFD. Mice were fed either an ND or HFD for 18 weeks, starting at 5 weeks of age. (a) Weekly weight measurements of the four groups of mice ( $n=8$ for ND and $n=12$ for HFD): E2-2.cre ${ }^{+} \mathrm{ND}$ (white triangles) E2-2.cre ${ }^{+} \mathrm{HFD}$ (black triangles), E2-2.cre ${ }^{-} \mathrm{ND}$ (white circles), E2-2.cre ${ }^{-}$HFD (black circles). (b) Fasting insulin levels for HFD-fed E22.cre ${ }^{+}$(white bar) and E2-2.cre ${ }^{-}$(black bar) mice. (c) An OGTT

pleiotropic cytokine family in several biological processes has also been proposed $[8,11]$. Type I IFNs have previously been indirectly implicated in obesity development based on the analysis of the IRF- $7^{-/-}$mouse [19]. IRF-7 plays an important role in innate immunity through the regulation of IFN- $\alpha / \beta$ secretion. These IFNs can further activate cells through interactions with IFNAR, thereby inducing additional IRF-7 expression, which is required for the type I IFN positive feedback loop. A study by Wang et al [19] suggests that improved energy expenditure protects IRF- $7^{-/}$mice from developing
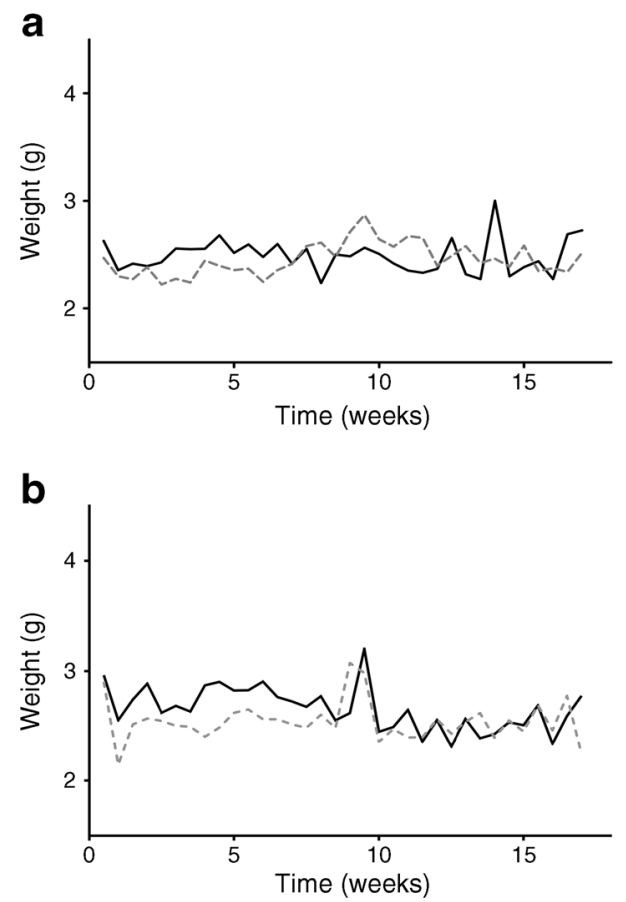

Fig. 5 Food intake of E2-2.cre ${ }^{+}$and IFNAR ${ }^{-/}$mice is unaltered compared with control mice. Food intake per cage for (a) IFNAR $^{-/}$(dashed grey line) and B6 mice (black line) and (b) E2-2.cre ${ }^{+}$(dashed grey line) and E2-2.cre (black line) littermates. $n=2-6$ cages, $n=6$ animals per cage. Two-way ANOVA was used; no significant differences were observed
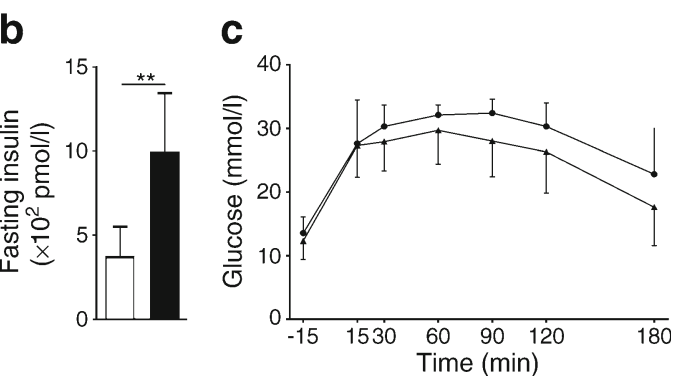

performed after 18 weeks $(n=6)$. Mice were given an oral dose of glucose $(2 \mathrm{~g} / \mathrm{kg})$ and blood glucose was determined from tail vein samples at different time points. E2-2.cre ${ }^{+}$HFD (black triangles), E2-2.cre HFD (black circles). The experiments were repeated independently 2-3 times. ${ }^{*} p \leq 0.01, * * * p \leq 0.001$ using two-way ANOVA $(\mathbf{a}, \mathbf{c})$ or Student's $t$ test (b)

DIO. In agreement with their findings, our data suggest that the protection from weight gain in the absence of type I IFN signalling is a consequence of altered immune-metabolism interplay, resulting in increased energy metabolism. This is in line with the notion that the immune system may play an important role in maintaining energy balance.

The pDCs are professional type I IFN producers responsible for the vast majority of IFN $\alpha / \beta$ secretion [29]. While pDCs are most well-known for their antiviral activity and ability to secrete vast amounts of IFN-I in response to TLR recognition of double-stranded RNA and $\mathrm{CpG}$ motifs, these cells have also been implicated in several disease conditions associated with systemic inflammation, including autoimmune diabetes [30, 31]. An abundance of nucleic acid ligands and a critical role of TLR-9 in obesity-associated inflammation has also been reported previously both in animal models and humans [22, 32-35]. The finding that IFNAR ${ }^{-/}$mice are protected from DIO highlights a possible role of pDCs in obesity-associated inflammation. While the pDCs have previously been found at an elevated frequency in the AT and liver of obese mice [4], human studies have found decreased [36-38] or unaltered [30,39] numbers of circulating pDCs in obese individuals with type 2 diabetes compared with lean individuals but have been unable to investigate the possible relocation of these cells. The data obtained from the analysis of the mouse model of DIO reported here and previously by Stefanovic-Racic et al [4] supports the notion of a recruitment and activation of pDCs in obese VAT possibly, as has been previously suggested, mediated by the adipokine chemerin $[22,40]$. However, in light of conflicting results [6], further studies are needed in order to elucidate this possibility.

The accumulation of pDCs in the liver during obesity development may suggest a role of this subset in this organ. Similar to the AT, the accumulation of pDCs in the liver was correlated with an increase in the number of proinflammatory macrophages. Even at steady state, pDCs are highly abundant in the liver, accounting for one-third of the DC population. 
While the precise reason for this accumulation is unknown, it is likely to be part of the requirement for the optimal defence mechanisms of the liver, as it encounters an array of substances through processed blood from the gastrointestinal tract. This includes gut-derived commensal bacterial products [41, 42], of which the composition is affected by what is ingested. As pDCs are known to play an important role in the defence against invading microbes, it is plausible that even a swift change in the nutrient composition could induce their TLR-mediated production of proinflammatory cytokines [43]. Indeed, studies have shown that the gut microbiome rapidly responds to dietary changes in both humans [44] and mice [45, 46], which has been proposed to contribute to obesity.

To directly address the pDCs as possible mediators of obesity development through their type I IFN-producing ability, we used a mouse with a conditional knockout of E2-2, the E22.cre ${ }^{+}$mouse, resulting in a specific block in the development of pDCs [23]. We found that these mice were protected from DIO development, comparable to the IFNAR ${ }^{-/}$mice. In addition, the mice were also protected from developing insulin resistance and showed mildly improved glucose tolerance. Taken together, our results suggest a key role for pDCderived type I IFN in obesity development. This concurs with, and gives genetic evidence for, a model recently presented by Ghosh et al [22] suggesting that low-grade inflammation associated with obesity is driven by type 1 IFN produced by pDCs recruited to VAT and leading to proinflammatory polarisation of adipose-resident macrophages.

Acknowledgements Thanks to B. Johansson-Lindbom and M. Dahlgren (Lund University, Sweden) for providing the $\mathrm{IFNAR}^{-/-}$mice Thanks to U. Axling (Lund University) for assisting in the use of the body composition scanner, and Å. Larefalk (Umeå University, Umeå, Sweden), A. Deronic and A.-C. Selberg (Lund University) for help with the DIO experiments.

Data availability All data generated or analysed during this study are included in this published article.

Funding This work was supported by grants from the Swedish research council, Novo Nordisk Foundation and the Lundberg Foundation.

Duality of interest No potential conflicts of interest relevant to this article were reported.

Contribution statement TDH, AS-C and DH designed the study, analysed the data and wrote the manuscript. TDH, AS-C, NF-P, LH and $\mathrm{JN}$ performed the experiments. All authors reviewed and approved the manuscript. DH is the guarantor of this work and, as such, had full access to all the data in the study and takes responsibility for the integrity of the data and the accuracy of the data analysis.

Open Access This article is distributed under the terms of the Creative Commons Attribution 4.0 International License (http:// creativecommons.org/licenses/by/4.0/), which permits unrestricted use, distribution, and reproduction in any medium, provided you give appropriate credit to the original author(s) and the source, provide a link to the Creative Commons license, and indicate if changes were made.

\section{References}

1. McNelis JC, Olefsky JM (2014) Macrophages, immunity, and metabolic disease. Immunity 41:36-48

2. Sica A, Mantovani A (2012) Macrophage plasticity and polarization: in vivo veritas. J Clin Invest 122:787-795

3. Dandona P, Aljada A, Bandyopadhyay A (2004) Inflammation: the link between insulin resistance, obesity and diabetes. Trends Immunol 25:4-7

4. Stefanovic-Racic M, Yang X, Turner MS et al (2012) Dendritic cells promote macrophage infiltration and comprise a substantial proportion of obesity-associated increases in CD11c+ cells in adipose tissue and liver. Diabetes 61:2330-2339

5. Patsouris D, Li PP, Thapar D, Chapman J, Olefsky JM, Neels JG (2008) Ablation of CD11c-positive cells normalizes insulin sensitivity in obese insulin resistant animals. Cell Metab 8:301-309

6. Bertola A, Ciucci T, Rousseau D et al (2012) Identification of adipose tissue dendritic cells correlated with obesity-associated insulin-resistance and inducing Th17 responses in mice and patients. Diabetes 61:2238-2247

7. Shi H, Kokoeva MV, Inouye K, Tzameli I, Yin H, Flier JS (2006) TLR4 links innate immunity and fatty acid-induced insulin resistance. J Clin Invest 116:3015-3025

8. Gordts PL, Foley EM, Lawrence R et al (2014) Reducing macrophage proteoglycan sulfation increases atherosclerosis and obesity through enhanced type I interferon signaling. Cell Metab 20:813826

9. Brierley MM, Fish EN (2002) Review: IFN-alpha/beta receptor interactions to biologic outcomes: understanding the circuitry. J Interferon Cytokine Res 22:835-845

10. Schoggins JW, Rice CM (2011) Interferon-stimulated genes and their antiviral effector functions. Curr Opin Virol 1:519-525

11. Abe M, Matsuda M, Kobayashi $\mathrm{H}$ et al (2008) Effects of statins on adipose tissue inflammation: their inhibitory effect on MyD88independent IRF3/IFN-beta pathway in macrophages. Arterioscler Thromb Vasc Biol 28:871-877

12. Pattison MJ, MacKenzie KF, Elcombe SE, Arthur JS (2013) IFNbeta autocrine feedback is required to sustain TLR induced production of MCP-1 in macrophages. FEBS Lett 587:1496-1503

13. Goossens P, Gijbels MJ, Zernecke A et al (2010) Myeloid type I interferon signaling promotes atherosclerosis by stimulating macrophage recruitment to lesions. Cell Metab 12:142-153

14. Weisberg SP, Hunter D, Huber R et al (2006) CCR2 modulates inflammatory and metabolic effects of high-fat feeding. J Clin Invest 116:115-124

15. Gough DJ, Messina NL, Clarke CJ, Johnstone RW, Levy DE (2012) Constitutive type I interferon modulates homeostatic balance through tonic signaling. Immunity 36:166-174

16. Gilliet M, Cao W, Liu YJ (2008) Plasmacytoid dendritic cells: sensing nucleic acids in viral infection and autoimmune diseases. Nat Rev Immunol 8:594-606

17. Honda K, Yanai H, Negishi H et al (2005) IRF-7 is the master regulator of type-I interferon-dependent immune responses. Nature 434:772-777

18. Lazear HM, Lancaster A, Wilkins $C$ et al (2013) IRF-3, IRF-5, and IRF-7 coordinately regulate the type I IFN response in myeloid dendritic cells downstream of MAVS signaling. PLoS Pathog 9: e1003118 
19. Wang XA, Zhang R, Zhang S et al (2013) Interferon regulatory factor 7 deficiency prevents diet-induced obesity and insulin resistance. Am J Physiol Endocrinol Metab 305:E485-E495

20. Padilla J, Jenkins NT, Thorne PK et al (2014) Identification of genes whose expression is altered by obesity throughout the arterial tree. Physiol Genomics 46:821-832

21. Kim SJ, Choi Y, Choi YH, Park T (2012) Obesity activates toll-like receptor-mediated proinflammatory signaling cascades in the adipose tissue of mice. J Nutr Biochem 23:113-122

22. Ghosh AR, Bhattacharya R, Bhattacharya S et al (2016) Adipose recruitment and activation of plasmacytoid dendritic cells fuel metaflammation. Diabetes 65:3440-3452

23. Cisse B, Caton ML, Lehner M et al (2008) Transcription factor- 2 is an essential and specific regulator of plasmacytoid dendritic cell development. Cell 135:37-48

24. Surwit RS, Kuhn CM, Cochrane C, McCubbin JA, Feinglos MN (1988) Diet-induced type II diabetes in C57BL/6J mice. Diabetes 37:1163-1167

25. Swiecki M, Colonna M (2010) Unraveling the functions of plasmacytoid dendritic cells during viral infections, autoimmunity, and tolerance. Immunol Rev 234:142-162

26. Yun TJ, Lee JS, Machmach K et al (2016) Indoleamine 2,3dioxygenase-expressing aortic plasmacytoid dendritic cells protect against atherosclerosis by induction of regulatory $t$ cells. Cell Metab 24:886

27. Krug A, French AR, Barchet W et al (2004) TLR9-dependent recognition of MCMV by IPC and DC generates coordinated cytokine responses that activate antiviral NK cell function. Immunity 21: $107-119$

28. Asselin-Paturel C, Brizard G, Chemin K et al (2005) Type I interferon dependence of plasmacytoid dendritic cell activation and migration. J Exp Med 201:1157-1167

29. Colonna M, Trinchieri G, Liu YJ (2004) Plasmacytoid dendritic cells in immunity. Nat Immunol 5:1219-1226

30. Allen JS, Pang K, Skowera A et al (2009) Plasmacytoid dendritic cells are proportionally expanded at diagnosis of type 1 diabetes and enhance islet autoantigen presentation to T cells through immune complex capture. Diabetes 58:138-145

31. Hansen L, Schmidt-Christensen A, Gupta S et al (2015) E2-2 dependent plasmacytoid dendritic cells control autoimmune diabetes. PLoS One 10:e0144090

32. Garcia-Martinez I, Santoro N, Chen Y et al (2016) Hepatocyte mitochondrial DNA drives nonalcoholic steatohepatitis by activation of TLR9. J Clin Invest 126:859-864
33. Nishimoto S, Fukuda D, Higashikuni Y et al (2016) Obesityinduced DNA released from adipocytes stimulates chronic adipose tissue inflammation and insulin resistance. Sci Adv 2:e1501332

34. Revelo XS, Ghazarian M, Chng MH et al (2016) Nucleic acidtargeting pathways promote inflammation in obesity-related insulin resistance. Cell Rep 16:717-730

35. Strissel KJ, Stancheva Z, Miyoshi H et al (2007) Adipocyte death, adipose tissue remodeling, and obesity complications. Diabetes 56 : 2910-2918

36. Blank SE, Johnson EC, Weeks DK, Wysham CH (2012) Circulating dendritic cell number and intracellular TNF-alpha production in women with type 2 diabetes. Acta Diabetol 49(Suppl 1): S25-S32

37. Seifarth CC, Hinkmann C, Hahn EG, Lohmann T, Harsch IA (2008) Reduced frequency of peripheral dendritic cells in type 2 diabetes. Exp Clin Endocrinol Diabetes 116:162-166

38. Summers KL, Marleau AM, Mahon JL, McManus R, Hramiak I, Singh B (2006) Reduced IFN-alpha secretion by blood dendritic cells in human diabetes. Clin Immunol 121:81-89

39. Musilli C, Paccosi S, Pala L et al (2011) Characterization of circulating and monocyte-derived dendritic cells in obese and diabetic patients. Mol Immunol 49:234-238

40. Ernst MC, Issa M, Goralski KB, Sinal CJ (2010) Chemerin exacerbates glucose intolerance in mouse models of obesity and diabetes. Endocrinology 151:1998-2007

41. Wu GD, Chen J, Hoffmann C et al (2011) Linking long-term dietary patterns with gut microbial enterotypes. Science 334:105-108

42. Tremaroli V, Backhed F (2012) Functional interactions between the gut microbiota and host metabolism. Nature 489:242-249

43. Ghanim H, Abuaysheh S, Sia CL et al (2009) Increase in plasma endotoxin concentrations and the expression of Toll-like receptors and suppressor of cytokine signaling-3 in mononuclear cells after a high-fat, high-carbohydrate meal: implications for insulin resistance. Diabetes Care 32:2281-2287

44. David LA, Maurice CF, Carmody RN et al (2014) Diet rapidly and reproducibly alters the human gut microbiome. Nature 505:559563

45. Turnbaugh PJ, Ridaura VK, Faith JJ, Rey FE, Knight R, Gordon JI (2009) The effect of diet on the human gut microbiome: a metagenomic analysis in humanized gnotobiotic mice. Sci Transl Med 1:6ra14

46. Faith JJ, McNulty NP, Rey FE, Gordon JI (2011) Predicting a human gut microbiota's response to diet in gnotobiotic mice. Science 333:101-104 